\title{
Aproximación a una definición de pensamiento computacional
}

\section{(Approach to a definition of computational thinking)}

\author{
Norailith Polanco Padrón \\ Sonia Ferrer Planchart \\ Mariana Fernández Reina \\ Universidad del Zulia (Venezuela)
}

DOI: http://dx.doi.org/10.5944/ried.24.1.27419

\section{Cómo referenciar este artículo:}

Polanco Padrón, N., Ferrer Planchart, S., y Fernández Reina, M. (2021). Aproximación a una definición de pensamiento computacional. RIED. Revista Iberoamericana de Educación a Distancia, 24(1), pp. 55-76. doi: http://dx.doi. org/10.5944/ried.24.1.27419

\section{Resumen}

El norte de este trabajo implica hacer eco sobre un término que se ha popularizado a lo largo de los años y cuyos orígenes tuvieron lugar desde la perspectiva técnica de las ciencias informáticas, para la automatización de información o la producción de sistemas, pero que hoy en día, se difunde por muchos profesionales de otras áreas y grupos interesados en comprender y adoptar un método diferente para pensar y asumir situaciones de vida, un concepto llamado pensamiento computacional. Esta investigación persigue el objetivo de contribuir con la construcción de la definición de pensamiento computacional, así como el conocimiento del término para fines formativos. La investigación es de tipo descriptiva con diseño documental, basada en la revisión de literatura relacionada con el tema, para especificar con propiedad la forma en la que el pensamiento computacional es visto como opción para la representación, adquisición de conocimientos y atención a problemas, desde la óptica de expertos como ISTE y CSTA (2011), Papert (1980), García-Peñalvo (2016), Wing (2006), entre otros. Se encontró que una definición consensuada sobre pensamiento computacional ha sido tema de constante debate dentro de la comunidad científica, y que una de las mejores opciones en torno a este pensamiento es su incorporación al currículo escolar obligatorio, desde etapas o niveles tempranos del sistema educativo, en orden de potenciar las destrezas analíticas en el infante a través de la aplicación de fundamentos informáticos.

Palabras clave: educación; formación; educación primaria; pensamiento computacional; procesos cognitivos. 


\begin{abstract}
The aim of this research revolves around a term that has become popular over the years and whose origins took place from the technical perspective of computer science in order to automate information or the production of systems. However, nowadays it is widely used by many professionals from several areas and groups that are interested in understanding and adopting a different way of thinking and assuming life situations. This concept is called Computational Thinking. This research pursues the objective of contributing to the construction of a definition on computational thinking, as well as to the use of this term for educational purposes. The research is descriptive with a documentary design, based on the review of literature related to the subject, in order to properly specify how computational thinking is seen as an option for the representation, acquisition of knowledge and attention to problems, from the viewpoint of experts as ISTE \& CSTA (2011), Papert (1980), García-Peñalvo (2016), Wing (2006), among others. It was found that a definition by consensus of computational thinking has been the subject of constant debate within the scientific community, and one of the best options surrounding this kind of thinking is its incorporation into the mandatory school curriculum, from early stages or levels of the educational system, in order to enhance the children's analytical skills through the application of computer principles.
\end{abstract}

Keywords: education; training; elementary school; computational thinking; cognitive processes.

Ciertas potencias mundiales y países en vías de desarrollo, han destacado una tendencia educativa consistente en activar reformas para la alfabetización de competencias y aptitudes digitales en el alumnado, que han sido establecidas dentro de los planes formativos en etapa de educación primaria, donde se toman en consideración muchas destrezas y saberes que resultan clave para la integralidad del ser, como pueden ser la lectura, escritura, habilidades matemáticas, creatividad, lógica, entre otras competencias; con especial ahínco en procesos cognitivos que le ceden al individuo una mejoría en su elasticidad mental para solventar problemas. El norte de este trabajo implica hacer eco sobre un término que se ha popularizado con el paso de los años y cuyos orígenes tuvieron lugar desde la perspectiva técnica de las ciencias informáticas, pero que hoy en día, se pregona por profesionales de otras áreas, así como grupos interesados en comprender y adoptar un método diferente para asumir las situaciones de vida, un concepto llamado pensamiento computacional.

Esta investigación persigue el objetivo de contribuir con la construcción de una definición sobre el pensamiento computacional, así como el conocimiento del término para fines formativos, con lo que se pueda reforzar en el estudiantado sus procesos de pensamiento para la búsqueda de soluciones ante problemas complejos. La misma se instituye desde la revisión de literaturas y documentos relacionados con el tema, para especificar con propiedad y sobre la óptica de expertos, la forma en 
la cual el pensamiento computacional es visto como opción para la representación, adquisición de conocimientos y atención a problemas.

El documento se estructura en distintos segmentos, queinician con la presentación de los primeros indicios en cuanto a la idea de pensar computacionalmente y las aproximaciones de Papert (1980) desarrolladas desde la década de los sesenta y setenta, luego se revisan los aportes teóricos del concepto. Por último, se ofrecen las discusiones y consideraciones finales que permiten soportar la información citada y se producen opiniones alternativas al respecto, donde se concibe al pensamiento computacional como un sistema de refuerzo ante las propias habilidades intelectuales de un aprendiz y por ende, a las soluciones lógicas que potencialmente podrán ser útiles para sus futuros proyectos de vida; razón que ha motivado esfuerzos hacia la consolidación de destrezas mentales superiores en niños y jóvenes, así como la enseñanza de codificación de programas.

\section{FUNDAMENTACIÓN TEÓRICA}

\section{Sobre los orígenes del pensamiento computacional}

Las primeras aproximaciones del pensamiento computacional pueden verse asociadas con la adquisición de herramientas cognitivas, que son usualmente instrumentadas mediante el desarrollo de sistemas dentro de los espacios educativos, donde, concretamente, el adiestramiento en áreas como programación y diseño de algoritmos, ha tenido desde una perspectiva mundial, un acrecentado auge en cuanto al currículo educativo. En efecto, la comprensión y empleo de técnicas computacionales, regularmente trabajadas mediante la escritura de códigos funcionales que permitan el procesamiento de datos y la resolución de cuestiones lógicas, resultan inquebrantablemente necesarias para la adquisición de competencias elementales para un aprendiz, que guardan relación con las estructuras y conexiones mentales conducentes a pensamientos de orden superior. Por ende, el estudio de la programación informática y algorítmica, como herramientas cognitivas para la vida, ha tenido ciertas permutas conceptuales durante años, y hoy por hoy, puede considerarse que tiene un identificador propio, denominado y ampliamente popularizado como pensamiento computacional (Wing, 2006).

Sin embargo, mucho antes de bautizar este enfoque de pensamiento, el matemático Seymour Papert hablaba durante las décadas de los sesenta y setenta, sobre algo que él llamaba pensamiento procedimental, término que para varios autores mantiene una estrecha semejanza con la más reciente denominación de este tipo de pensamiento, ya que en sus ideas, manifestaba abiertamente la inclusión de procedimientos de desarrollo, representación, testeo y depuración, así como un efectivo procedimiento paso a paso, con el que una persona fuese capaz de abordar un problema. 
$\mathrm{Al}$ respecto, resulta fundamental destacar que Papert (1980), co-fundador del Laboratorio de Inteligencia Artificial del Instituto Tecnológico de Massachusetts (MIT); es para muchos, el padre del pensamiento computacional, dado a la derivación de sus trabajos con lenguajes de programación visual, desarrollados desde los años sesenta. Dentro de la comunidad científica en el área de la computación, Papert es conocido por ser el creador del lenguaje de programación de alto nivel LOGO, un lenguaje funcional, estructurado y puntualmente, de fácil aprendizaje, porque su finalidad se inclinaba a la obtención de resultados educativos.

Por tanto, es notable que, desde hace décadas, se empezaba a considerar una formación especializada para los jóvenes estudiantes, una instrucción que conformemente involucrara disciplinas matemáticas y lógicas. Durante años posteriores, el debate y la curiosidad sobre estas ideas no han cesado, y desde luego, aunado a la convergencia tecnológica y las nuevas reformas sociales, basadas en aptitudes y conocimientos, el término ha mutado y ha dado pie a continuas reflexiones sobre la búsqueda de una definición formal, que abarque de lo que realmente se trata ser un pensador computacional, cuya implicación supere la base de concebirlo como una destreza cardinal para la producción de aplicaciones de software, manejo de lenguajes de programación o para el diseño de algoritmos únicamente, debe ser visto más bien, como una competencia medular con la que se fortalezcan los modelos de la mente humana, para lograr mejores soluciones en cualquier rubro del saber universal. De esta forma y propiamente, la primera publicación de este enfoque, tuvo lugar en 2006 bajo la tutela de Wing, quien lo defendía como una competencia a ser tomada en cuenta durante la formación de los niños y lo denominaba pensamiento computacional.

\section{El concepto de pensamiento computacional}

La definición formal de pensamiento computacional o Computational Thinking fue introducida por vez primera dentro de la comunidad científica en computación, por Wing (2006), quien define el término como una forma de pensar que no se restringe en exclusiva hacia programadores de sistemas ni científicos en computación, sino como un grupo de habilidades útiles para todas las personas. Esta definición incluye a su vez, un considerable rango de herramientas mentales que reflejan y ponen en manifiesto la amplitud del campo potencial individual.

Su concepción del pensamiento computacional como un grupo de herramientas mentales con las que toda persona debería contar y aplicar para solventar situaciones, es un punto bastante natural, pero adiciona el hecho de diseñar sistemas y comprender el comportamiento humano. Esta primera definición ha sido mundialmente conocida por defender la idea sobre la incorporación del pensamiento computacional en la educación de todo ser humano, lo que, sin duda, tuvo -y tiene- un significativo impacto para el profesorado, tanto en áreas como ciencias de la computación, como en cualquier rama disciplinaria. 
Sucesivamente, otros expertos han realizado sus aportes a la definición del término, intentando esclarecerlo y establecer una definición consensuada, la cual pueda ser aceptada por la comunidad científica que desarrolla el tema, sin embargo, esta meta no se ha alcanzado. Los nuevos desarrollos tecnológicos y los análisis al respecto de las competencias de alfabetización digital, promueven el surgimiento de nuevos elementos relacionados con el pensamiento computacional, y, pese a que se han desarrollado proyectos para su introducción en el currículo de distintos niveles educativos, no existe una definición formal para el término, por lo cual estas iniciativas son variadas en cuanto a contenido y naturaleza (García-Peñalvo, 2016).

Por tal motivo, en este documento se ha realizado un recorrido por distintas definiciones que han surgido sobre el pensamiento computacional desde que este fue introducido por Wing en 2006, hasta algunos más actuales que destacan distintos énfasis y procesos mentales implicados en su desarrollo y consolidación.

\section{METODOLOGÍA}

Según su objetivo, esta investigación, se corresponde con el tipo descriptivo, siguiendo un diseño documental, dado que fuentes utilizadas para la obtención de la información estuvieron constituidas por textos resultantes de estudios previos. El procedimiento documental se estableció en pasos consecutivos que iniciaron con la selección y delimitación del tema, seguido de la recolección de literaturas referidas a la conceptualización del pensamiento computacional. Desde autores pioneros en el área, hasta investigaciones más recientes, se efectuó la consulta a diversas fuentes bibliográficas impresas y electrónicas, para luego organizar y tabular la información recabada, y con base en esto discutir los resultados.

En cuanto a la cantidad de documentos revisados, esta asciende a más de 100, entre fuentes impresas y electrónicas, artículos en revistas, libros, tesis doctorales, trabajos de grado y páginas web, de las cuales se extrajeron las definiciones que se sistematizan en el siguiente apartado.

\section{RESULTADOS Y DISCUSIÓN}

Los resultados obtenidos de la revisión y el análisis documental para la extracción de las definiciones sobre pensamiento computacional, se organizan cronológica y alfabéticamente en la siguiente tabla, comenzando por la ofrecida por Wing (2006) considerada la primera definición formal, hasta las más actuales que se han considerado. 
Tabla 1. Definiciones del término pensamiento computacional

\begin{tabular}{|c|c|c|c|}
\hline No & Autor & Definición & Énfasis \\
\hline 1 & $\begin{array}{l}\text { Wing } \\
(2006)\end{array}$ & $\begin{array}{l}\text { El pensamiento computacional implica resolver } \\
\text { los problemas, diseñar sistemas y entender el } \\
\text { comportamiento humano, aprovechando los } \\
\text { conceptos fundamentales para las ciencias } \\
\text { informáticas. El pensamiento computacional incluye } \\
\text { una gama de herramientas mentales que reflejan la } \\
\text { amplitud del campo de la informática. }\end{array}$ & $\begin{array}{c}\text { Herramientas } \\
\text { mentales }\end{array}$ \\
\hline 2 & $\begin{array}{l}\text { Cuny, Snyder y } \\
\text { Wing } \\
(2010)\end{array}$ & $\begin{array}{l}\text { Actividad mental para formular problemas de } \\
\text { forma que admitan una solución computacional; } \\
\text { el pensamiento computacional es el proceso de } \\
\text { pensamiento involucrado en la formulación de un } \\
\text { problema y la expresión de sus soluciones de tal } \\
\text { manera que una computadora, humano o máquina, } \\
\text { puede llevar a cabo eficazmente. }\end{array}$ & $\begin{array}{c}\text { Actividad } \\
\text { mental }\end{array}$ \\
\hline 3 & $\begin{array}{l}\text { Wing } \\
(2010)\end{array}$ & $\begin{array}{l}\text { El pensamiento computacional son los procesos } \\
\text { de pensamiento involucrados en la formulación de } \\
\text { problemas y sus soluciones para que las soluciones } \\
\text { se representen en una forma que pueda ser llevada a } \\
\text { cabo efectivamente por un agente de procesamiento } \\
\text { de información. }\end{array}$ & $\begin{array}{l}\text { Procesos de } \\
\text { pensamiento }\end{array}$ \\
\hline 4 & $\begin{array}{l}\text { Barr y } \\
\text { Stephenson } \\
\text { (2011) }\end{array}$ & $\begin{array}{l}\text { Es un proceso de resolución de problemas } \\
\text { que incluye (pero no se limita a) las siguientes } \\
\text { características: formular problemas de una manera } \\
\text { que nos permita usar una computadora y otras } \\
\text { herramientas para ayudar. }\end{array}$ & $\begin{array}{l}\text { Proceso de } \\
\text { resolución de } \\
\text { problemas }\end{array}$ \\
\hline 5 & $\begin{array}{l}\text { ISTE y CSTA } \\
(2011)\end{array}$ & $\begin{array}{l}\text { Es un proceso para dar resolución a los problemas. } \\
\text { El pensamiento computacional es un enfoque para } \\
\text { resolver un determinado problema que empodera } \\
\text { la integración de tecnologías digitales con ideas } \\
\text { humanas. No reemplaza el énfasis en creatividad, } \\
\text { razonamiento o pensamiento crítico pero refuerza } \\
\text { esas habilidades al tiempo que realza formas de } \\
\text { organizar el problema de manera que el computador } \\
\text { pueda ayudar. }\end{array}$ & $\begin{array}{l}\text { Proceso de } \\
\text { resolución de } \\
\text { problemas } \\
\text { Enfoque }\end{array}$ \\
\hline 6 & $\begin{array}{l}\text { Brennan } \\
(2011)\end{array}$ & $\begin{array}{l}\text { Conjunto de conceptos, prácticas y perspectivas } \\
\text { basadas en las precisiones del mundo de la } \\
\text { informática. }\end{array}$ & Dimensiones \\
\hline 7 & $\begin{array}{l}\text { Aho } \\
(2012)\end{array}$ & $\begin{array}{l}\text { Es un conjunto de procesos de pensamiento } \\
\text { involucrados en la formulación de problemas para } \\
\text { que sus soluciones se puedan representar como pasos } \\
\text { y algoritmos computacionales. }\end{array}$ & $\begin{array}{l}\text { Procesos de } \\
\text { pensamiento }\end{array}$ \\
\hline
\end{tabular}




\begin{tabular}{|c|c|c|c|}
\hline No & Autor & Definición & Énfasis \\
\hline 8 & $\begin{array}{l}\text { The Royal } \\
\text { Society } \\
(2012)\end{array}$ & $\begin{array}{l}\text { El pensamiento computacional es el proceso de } \\
\text { reconocimiento de aspectos de la informática en } \\
\text { el mundo que nos rodea, y aplicar herramientas y } \\
\text { técnicas de la informática para comprender y razonar } \\
\text { sobre los sistemas y procesos tanto naturales como } \\
\text { artificiales. }\end{array}$ & $\begin{array}{c}\text { Proceso de } \\
\text { pensamiento }\end{array}$ \\
\hline 9 & $\begin{array}{c}\text { Selby y Wollard } \\
(2013)\end{array}$ & $\begin{array}{l}\text { El pensamiento computacional es una actividad, a } \\
\text { menudo orientada a un producto, asociada con la } \\
\text { resolución de problemas, pero no limitada a ésta, que } \\
\text { a su vez consagra un grupo selecto de habilidades, } \\
\text { a saber: habilidad para pensar en abstracciones, } \\
\text { pensar en términos de descomposición, pensar de } \\
\text { forma algorítmica, pensar en términos de evaluación } \\
\text { y pensar en generalizaciones. }\end{array}$ & $\begin{array}{l}\text { Actividad } \\
\text { mental }\end{array}$ \\
\hline 10 & $\begin{array}{c}\text { Sysło y } \\
\text { Kwiatkowska } \\
(2013)\end{array}$ & $\begin{array}{l}\text { El pensamiento computacional es un conjunto de } \\
\text { habilidades de pensamiento que pueden no resultar } \\
\text { en programación de computadoras, debería enfocarse } \\
\text { en los principios de la computación más que en las } \\
\text { habilidades de programación de computadoras. }\end{array}$ & $\begin{array}{c}\text { Habilidades } \\
\text { de } \\
\text { pensamiento }\end{array}$ \\
\hline 11 & $\begin{array}{c}\text { Mannila, } \\
\text { Dagiene, Demo, } \\
\text { Grgurina, } \\
\text { Mirolo, } \\
\text { Rolandsson y } \\
\text { Settle } \\
(2014) \\
\end{array}$ & $\begin{array}{l}\text { El pensamiento computacional es un término } \\
\text { que abarca un conjunto de conceptos y procesos } \\
\text { de pensamiento de la informática que ayudan a } \\
\text { formular problemas y sus soluciones en diferentes } \\
\text { disciplinas. }\end{array}$ & $\begin{array}{l}\text { Procesos de } \\
\text { pensamiento }\end{array}$ \\
\hline 12 & $\begin{array}{l}\text { Raja } \\
(2014)\end{array}$ & $\begin{array}{l}\text { El pensamiento computacional se basa en ver el } \\
\text { mundo como una serie de puzzles, que pueden } \\
\text { romperse en trozos más pequeños y ser resuelto poco } \\
\text { a poco, al hacer uso de la lógica y el razonamiento } \\
\text { deductivo. }\end{array}$ & $\begin{array}{l}\text { Visión del } \\
\text { mundo }\end{array}$ \\
\hline 13 & $\begin{array}{c}\text { Basogain, Olabe } \\
\text { y Olabe } \\
(2015)\end{array}$ & $\begin{array}{l}\text { El pensamiento computacional es una metodología } \\
\text { basada en la implementación de los conceptos } \\
\text { básicos de las ciencias de la computación para } \\
\text { resolver problemas cotidianos, diseñar sistemas } \\
\text { domésticos y realizar tareas rutinarias. Esta nueva } \\
\text { forma de abordar los problemas permite resolver con } \\
\text { eficacia y éxito problemas que de otra forma no son } \\
\text { tratables por una persona. }\end{array}$ & Metodología \\
\hline 14 & $\begin{array}{c}\text { Computing at } \\
\text { School (CAS) } \\
\quad(2015)\end{array}$ & $\begin{array}{l}\text { El pensamiento computacional es un proceso } \\
\text { cognitivo o de pensamiento que implica el } \\
\text { razonamiento lógico por el cual se resuelven los } \\
\text { problemas y se entienden mejor los artefactos, } \\
\text { procedimientos y sistemas. }\end{array}$ & $\begin{array}{c}\text { Proceso de } \\
\text { pensamiento }\end{array}$ \\
\hline
\end{tabular}




\begin{tabular}{|c|c|c|c|}
\hline $\mathbf{N}^{\mathbf{O}}$ & Autor & Definición & Énfasis \\
\hline 15 & $\begin{array}{l}\text { Pérez y Roig- } \\
\text { Vila } \\
(2015)\end{array}$ & $\begin{array}{l}\text { El pensamiento computacional es un tipo de } \\
\text { pensamiento que se relaciona con el pensamiento } \\
\text { matemático, lógico y crítico, en el que intervienen } \\
\text { habilidades como el reconocimiento de patrones, } \\
\text { abstracción y modelación; cuya finalidad es proponer } \\
\text { soluciones a los problemas reales de la vida cotidiana } \\
\text { aplicando herramientas informáticas. }\end{array}$ & $\begin{array}{l}\text { Habilidades } \\
\text { del } \\
\text { pensamiento } \\
\text { Algoritmos }\end{array}$ \\
\hline 16 & Snalune (2015) & $\begin{array}{l}\text { El pensamiento computacional como aquellos } \\
\text { procesos de pensamiento involucrados en la } \\
\text { resolución de problemas, que integran habilidades } \\
\text { importantes para que el estudiante pueda contribuir } \\
\text { con el entorno laboral competitivo. }\end{array}$ & $\begin{array}{c}\text { Procesos de } \\
\text { pensamiento }\end{array}$ \\
\hline 17 & $\begin{array}{l}\text { Cummins } \\
(2016)\end{array}$ & $\begin{array}{l}\text { El pensamiento computacional es un método } \\
\text { estructurado y probado diseñado para identificar } \\
\text { problemas independientemente de la edad o el nivel } \\
\text { de alfabetización informática. }\end{array}$ & Método \\
\hline 18 & $\begin{array}{c}\text { García-Peñalvo } \\
(2016)\end{array}$ & $\begin{array}{l}\text { El pensamiento computacional consiste en la } \\
\text { aplicación de un alto nivel de abstracción y un } \\
\text { enfoque algorítmico para resolver cualquier tipo de } \\
\text { problemas. }\end{array}$ & Actividad \\
\hline 19 & $\begin{array}{l}\text { García-Peñalvo, } \\
\text { Reimann, } \\
\text { Tuul, Rees y } \\
\text { Jormanainen } \\
\text { (2016) }\end{array}$ & $\begin{array}{l}\text { El pensamiento computacional es principalmente } \\
\text { una metodología activa de resolución de problemas } \\
\text { en los estudiantes; utiliza un conjunto de conceptos, } \\
\text { como abstracción o iteración entre otros, para } \\
\text { procesar y analizar datos, y para crear artefactos } \\
\text { reales o virtuales. }\end{array}$ & $\begin{array}{c}\text { Metodología } \\
\text { activa }\end{array}$ \\
\hline 20 & $\begin{array}{l}\text { Román } \\
\text { (2016) }\end{array}$ & $\begin{array}{l}\text { El pensamiento computacional sería un término de } \\
\text { corte cognitivo; se trata de un emergente constructo } \\
\text { psicológico que se puede definir como la capacidad } \\
\text { de formular y solucionar problemas apoyándose } \\
\text { en conceptos computacionales (secuencias, bucles, } \\
\text { condicionales, funciones, variables), y siguiendo } \\
\text { la lógica algorítmica inherente a los lenguajes } \\
\text { informáticos de programación. }\end{array}$ & $\begin{array}{l}\text { Capacidad } \\
\text { para resolver } \\
\text { problemas }\end{array}$ \\
\hline 21 & $\begin{array}{l}\text { Formación en } \\
\text { Red del INTEF } \\
\text { (s.f.) }\end{array}$ & $\begin{array}{l}\text { Proceso mental, y por tanto humano en el cual se } \\
\text { piensa en las ideas y se combinan con datos mediante } \\
\text { las TIC para resolver problemas. }\end{array}$ & $\begin{array}{c}\text { Proceso } \\
\text { mental }\end{array}$ \\
\hline 22 & $\begin{array}{l}\text { Plan Ceibal } \\
(2017)\end{array}$ & $\begin{array}{l}\text { El pensamiento computacional es una manera de } \\
\text { pensar que no se restringe al código, la programación } \\
\text { y la computadora, en la cual se emplea razonamiento } \\
\text { lógico, pensamiento algorítmico y técnicas de } \\
\text { resolución de problemas, y se involucran la } \\
\text { creatividad, la habilidad de diseño y la resolución de } \\
\text { problemas. }\end{array}$ & $\begin{array}{l}\text { Manera de } \\
\text { pensar }\end{array}$ \\
\hline
\end{tabular}




\begin{tabular}{|c|c|c|c|}
\hline $\mathbf{N}^{\mathbf{0}}$ & Autor & Definición & Énfasis \\
\hline 23 & $\begin{array}{l}\text { Llorens, García- } \\
\text { Peñalvo, Molero } \\
\text { y Vendrell } \\
\text { (2017) }\end{array}$ & $\begin{array}{l}\text { El pensamiento computacional es un paradigma de } \\
\text { trabajo para la resolución de casos y la programación } \\
\text { informática como herramienta para atender estos } \\
\text { problemas, por tanto, este pensamiento dota al } \\
\text { individuo de las herramientas cognitivas necesarias } \\
\text { para desenvolverse con éxito en el mundo digital en } \\
\text { el que se desenvuelven. }\end{array}$ & $\begin{array}{c}\text { Paradigma de } \\
\text { trabajo }\end{array}$ \\
\hline 24 & $\begin{array}{l}\text { Ortega } \\
(2017)\end{array}$ & $\begin{array}{l}\text { El pensamiento computacional se traduce en una } \\
\text { estrategia computacional que ayuda a resolver } \\
\text { problemas complejos, al permitir la reformulación de } \\
\text { eventos en términos más sencillos y manejables para } \\
\text { quien piensa. }\end{array}$ & Estrategia \\
\hline 25 & $\begin{array}{l}\text { Shute, Sun y } \\
\text { Asbell-Clarke } \\
\quad(2017)\end{array}$ & $\begin{array}{l}\text { El pensamiento computacional es una forma de } \\
\text { pensar para resolver problemas de manera efectiva } \\
\text { y eficiente (es decir, algorítmicamente, con o sin la } \\
\text { ayuda de computadoras) con soluciones que son } \\
\text { reutilizables en diferentes contextos. }\end{array}$ & $\begin{array}{l}\text { Forma de } \\
\text { pensar }\end{array}$ \\
\hline 26 & $\begin{array}{l}\text { Rodríguez } \\
\text { (2018) }\end{array}$ & $\begin{array}{l}\text { Proceso de pensamiento a través del cual una } \\
\text { persona plantea un problema y su posible solución o } \\
\text { soluciones de tal manera que este pueda ser resuelto } \\
\text { utilizando una secuencia de instrucciones ejecutadas } \\
\text { por un humano, una computadora o ambos. Es } \\
\text { utilizado para resolver problemas de distintas } \\
\text { disciplinas como pueden ser las matemáticas, } \\
\text { biología, humanidades y otras. }\end{array}$ & $\begin{array}{l}\text { Proceso } \\
\text { mental }\end{array}$ \\
\hline 27 & $\begin{array}{l}\text { Codelearn } \\
(2019)\end{array}$ & $\begin{array}{l}\text { El pensamiento computacional es un proceso mental } \\
\text { que lleva al individuo a buscar soluciones óptimas, } \\
\text { eficientes y abiertas. No solamente aplicable al } \\
\text { mundo informático. }\end{array}$ & $\begin{array}{c}\text { Proceso } \\
\text { mental }\end{array}$ \\
\hline 28 & $\begin{array}{l}\text { González- } \\
\text { González } \\
\text { (2019) }\end{array}$ & $\begin{array}{l}\text { El pensamiento computacional es la aproximación } \\
\text { hacia la resolución de problemas mediante el uso } \\
\text { de determinadas estrategias, que a su vez implica } \\
\text { formular problemas de una manera que permite el } \\
\text { uso de un computador para resolverlos; organizando } \\
\text { y analizando lógicamente los datos trabajados, } \\
\text { además, estos son representados a través de } \\
\text { abstracciones, automatizando soluciones a través de } \\
\text { algoritmos. }\end{array}$ & Estrategias \\
\hline 29 & $\begin{array}{l}\text { Maris } \\
(2019)\end{array}$ & $\begin{array}{l}\text { Se denomina pensamiento computacional a un tipo } \\
\text { de pensamiento analítico, que puede ser desarrollado } \\
\text { y aplicado en distintas disciplinas o actividades de la } \\
\text { vida cotidiana. }\end{array}$ & $\begin{array}{c}\text { Tipo de } \\
\text { pensamiento }\end{array}$ \\
\hline
\end{tabular}




\begin{tabular}{|c|c|c|c|}
\hline No & Autor & Definición & Énfasis \\
\hline 30 & $\begin{array}{c}\text { Zapata-Ros } \\
\text { (2019) }\end{array}$ & $\begin{array}{l}\text { El pensamiento computacional desenchufado } \\
\text { (Computational Thinking Unplugged) hace } \\
\text { referencia al conjunto de actividades, y a su diseño } \\
\text { educativo, que se planean y utilizan para fomentar } \\
\text { en los niños, desde primeras etapas de su desarrollo } \\
\text { cognitivo (educación infantil, primer tramo de la } \\
\text { educación primaria, juegos en casa con los padres } \\
\text { y los amigos,) habilidades que luego pueden ser } \\
\text { evocadas para potenciar un buen aprendizaje del } \\
\text { pensamiento computacional en otras etapas, o } \\
\text { en la formación técnica, profesional incluso en la } \\
\text { educación universitaria. }\end{array}$ & $\begin{array}{c}\text { Actividades y } \\
\text { habilidades }\end{array}$ \\
\hline
\end{tabular}

En la tabla 1 pueden apreciarse 30 definiciones extraídas de las distintas fuentes examinadas, publicadas durante las últimas décadas, de las cuales pudo inferirse el enfoque de cada autor acerca del pensamiento computacional a partir del elemento en el cual pone el énfasis de su definición. En cuanto a los años en los cuales fueron publicadas estas definiciones, se encuentra que la primera en aparecer es la propuesta por Wing (2006). Según las fuentes consultadas, es a partir de 2010 cuando el término pensamiento computacional cobra fuerza y desde entonces surgen nuevas concepciones, siendo los últimos años los más prolíficos en cuanto a la definición del mismo. La figura 1 muestra cómo se distribuyen las definiciones seleccionadas en esta investigación entre los años 2006 a 2019.

Figura 1. Distribución de las definiciones seleccionadas según el año

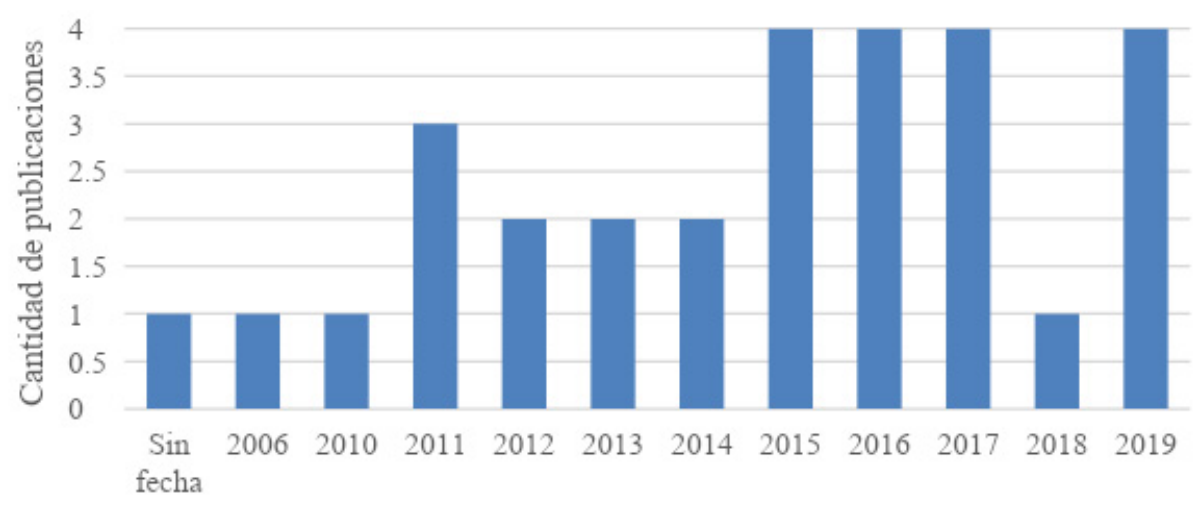

\section{Año de publicación}

Fuente: Tabla 1. 
Adicionalmente, la mayoría de las definiciones seleccionadas han surgido en España (11) y Estados Unidos (10). También se ha estudiado el tema en Reino Unido (4), Latinoamérica (3) y otros países europeos (2), generalmente en el ámbito universitario y en organizaciones que apoyan la formación informática en general. En la siguiente tabla se resumen las características de las definiciones según la cantidad de autores que la suscriben, así como la nacionalidad y adscripción del autor principal de cada una.

Tabla 2. Características de las definiciones seleccionadas

\begin{tabular}{|c|c|c|c|}
\hline Autores & $\begin{array}{l}\text { Cantidad } \\
\text { de autores }\end{array}$ & $\begin{array}{c}\text { País del autor } \\
\text { principal }\end{array}$ & Filiación \\
\hline $\begin{array}{l}\text { Wing } \\
(2006)\end{array}$ & 1 & USA & $\begin{array}{l}\text { Carnegie Mellon } \\
\text { University }\end{array}$ \\
\hline $\begin{array}{l}\text { Cuny, Snyder y Wing } \\
\text { (2010) }\end{array}$ & 3 & USA & National Science Found \\
\hline $\begin{array}{l}\text { Wing } \\
(2010)\end{array}$ & 1 & USA & $\begin{array}{l}\text { Carnegie Mellon } \\
\text { University }\end{array}$ \\
\hline Barr y Stephenson (2011) & 2 & USA & Union College \\
\hline $\begin{array}{l}\text { ISTE y CSTA } \\
(2011)\end{array}$ & 2 & USA & ISTE \\
\hline $\begin{array}{c}\text { Brennan } \\
(2011)\end{array}$ & 1 & USA & Harvard University \\
\hline $\begin{array}{l}\text { Aho } \\
(2012)\end{array}$ & 1 & USA & Columbia University \\
\hline $\begin{array}{l}\text { The Royal Society } \\
\text { (2012) }\end{array}$ & Institucional & Reino Unido & The Royal Society \\
\hline $\begin{array}{l}\text { Selby y Wollard } \\
(2013)\end{array}$ & 2 & Reino Unido & $\begin{array}{l}\text { Universidad de } \\
\text { Southampton }\end{array}$ \\
\hline $\begin{array}{c}\text { Sysło y Kwiatkowska } \\
(2013)\end{array}$ & 2 & Polonia & $\begin{array}{c}\text { Nicolaus Copernicus } \\
\text { University }\end{array}$ \\
\hline $\begin{array}{l}\text { Mannila, Dagiene, Demo, } \\
\text { Grgurina, Mirolo, Rolandsson } \\
\text { y Settle } \\
(2014)\end{array}$ & 7 & Finlandia & Åbo Akademi University \\
\hline $\begin{array}{c}\text { Raja } \\
(2014)\end{array}$ & 1 & USA & Periodista independiente \\
\hline $\begin{array}{l}\text { Basogain, Olabe y Olabe } \\
\text { (2015) }\end{array}$ & 3 & España & Universidad del País Vasco \\
\hline $\begin{array}{l}\text { Computing at School (CAS) } \\
(2015)\end{array}$ & 1 & Reino Unido & Computing at school \\
\hline
\end{tabular}




\begin{tabular}{|c|c|c|c|}
\hline Autores & $\begin{array}{l}\text { Cantidad } \\
\text { de autores }\end{array}$ & $\begin{array}{c}\text { País del autor } \\
\text { principal }\end{array}$ & Filiación \\
\hline $\begin{array}{l}\text { Pérez y Roig-Vila } \\
\text { (2015) }\end{array}$ & 2 & Ecuador & Universidad de Ecuador \\
\hline $\begin{array}{l}\text { Snalune } \\
(2015)\end{array}$ & 1 & Reino Unido & Codio \\
\hline $\begin{array}{l}\text { Cummins } \\
\text { (2016) }\end{array}$ & 1 & USA & Edagalaxy.com \\
\hline $\begin{array}{l}\text { García-Peñalvo } \\
(2016)\end{array}$ & 1 & España & Universidad de Salamanca \\
\hline $\begin{array}{l}\text { García-Peñalvo, Reimann, } \\
\text { Tuul, Rees, y Jormanainen } \\
\text { (2016) }\end{array}$ & 5 & España & Universidad de Salamanca \\
\hline $\begin{array}{l}\text { Román } \\
\text { (2016) }\end{array}$ & 1 & España & $\begin{array}{l}\text { Universidad Nacional de } \\
\text { Educación a Distancia }\end{array}$ \\
\hline $\begin{array}{l}\text { Formación en Red del INTEF } \\
\text { (s.f.) }\end{array}$ & Institucional & España & INTEF \\
\hline $\begin{array}{l}\text { Plan Ceibal } \\
(2017)\end{array}$ & Institucional & Uruguay & $\begin{array}{l}\text { Administración Nacional } \\
\text { de Educación Pública }\end{array}$ \\
\hline $\begin{array}{c}\text { Llorens, García-Peñalvo, } \\
\text { Molero y Vendrell } \\
(2017) \\
\end{array}$ & 4 & España & Universidad de Alicante \\
\hline $\begin{array}{l}\text { Ortega } \\
(2017)\end{array}$ & 1 & España & $\begin{array}{c}\text { Universidad Internacional } \\
\text { de La Rioja }\end{array}$ \\
\hline $\begin{array}{l}\text { Shute, Dun y } \\
\text { Asbell-Clarke } \\
\quad(2017)\end{array}$ & 3 & USA & $\begin{array}{l}\text { Universidad Estatal de } \\
\text { Florida }\end{array}$ \\
\hline $\begin{array}{l}\text { Rodríguez } \\
\text { (2018) }\end{array}$ & 1 & España & Elurnet \\
\hline $\begin{array}{l}\text { Codelearn } \\
(2019)\end{array}$ & Institucional & España & Codelearn \\
\hline $\begin{array}{l}\text { González-González } \\
\text { (2019) }\end{array}$ & 1 & España & Universidad de La Laguna \\
\hline $\begin{array}{l}\text { Maris } \\
(2019)\end{array}$ & 1 & Argentina & Net-learning \\
\hline $\begin{array}{l}\text { Zapata-Ros } \\
\text { (2019) }\end{array}$ & 1 & España & Universidad de Murcia \\
\hline
\end{tabular}

Por otra parte, el análisis de las definiciones permitió identificar al menos ocho criterios de énfasis, facilitando su agrupamiento de acuerdo a estos. Cabe destacar que, cada uno de estos criterios tiene implicaciones particulares y perspectivas de aplicación en la educación, así como en la investigación por la cual los autores 
construyen el conocimiento acerca del pensamiento computacional. Estos resultados se presentan a continuación.

Tabla 3. Énfasis de las definiciones e implicaciones educativas

\begin{tabular}{|c|c|c|}
\hline Autores & Énfasis & Perspectiva educativa \\
\hline $\begin{array}{l}\text { Wing } \\
(2006)\end{array}$ & $\begin{array}{l}\text { Herramientas } \\
\text { mentales }\end{array}$ & $\begin{array}{l}\text { Se entiende como una cualidad que todas } \\
\text { las personas deben poseer, para resolver } \\
\text { problemas y diseñar sistemas, por lo tanto, } \\
\text { el pensamiento computacional debería ser } \\
\text { incluido como una nueva competencia en } \\
\text { la formación educativa porque, al igual que } \\
\text { la matemática u otra disciplina del saber, es } \\
\text { una habilidad fundamental cuya progresión } \\
\text { en la comprensión de un concepto se basa } \\
\text { en la comprensión del anterior y se puede } \\
\text { desarrollar desde edades tempranas. }\end{array}$ \\
\hline $\begin{array}{c}\text { Cuny et al. (2010) } \\
\text { Wing (2010) } \\
\text { Aho (2012) } \\
\text { The Royal Society } \\
\text { (2012) } \\
\text { Selby y Wollard (2013) } \\
\text { Mannila et al. (2014) } \\
\text { Computing at school } \\
\text { (2015) } \\
\text { Snalune (2015) } \\
\text { García-Peñalvo (2016) } \\
\text { Formación en red } \\
\text { (INTEF, S.F.) } \\
\text { Rodríguez (2018) } \\
\text { Codelearn (2019) }\end{array}$ & $\begin{array}{l}\text { Proceso mental } \\
\text { Actividad mental }\end{array}$ & $\begin{array}{l}\text { Indistintamente a lo que se dedique } \\
\text { posteriormente un niño, seguro es que } \\
\text { tendrá que saber utilizar herramientas } \\
\text { tecnológicas tanto para su trabajo como para } \\
\text { interactuar en su vida cotidiana. Por lo tanto, } \\
\text { es imprescindible que se eduque a los niños } \\
\text { y niñas para que sean capaces de hacer que } \\
\text { las máquinas hagan lo que ellos quieran y no } \\
\text { al revés. } \\
\text { Los beneficios educativos del pensamiento } \\
\text { computacional son varios, incluyen el uso } \\
\text { de abstracciones que mejoran y refuerzan } \\
\text { las habilidades intelectuales, y que por tanto } \\
\text { pueden ser transferidas a cualquier otro } \\
\text { ámbito. } \\
\text { Los informáticos ya conocen el valor del } \\
\text { pensamiento abstracto para manejar la } \\
\text { complejidad. }\end{array}$ \\
\hline $\begin{array}{l}\text { Barr y Stephenson } \\
\text { (2011) } \\
\text { ISTE Y CSTA (2011) }\end{array}$ & $\begin{array}{l}\text { Proceso de } \\
\text { resolución de } \\
\text { problemas }\end{array}$ & $\begin{array}{l}\text { Los estudiantes al programar y compartir } \\
\text { proyectos, comienzan a desarrollarse como } \\
\text { pensadores computacionales, al aprender } \\
\text { nociones y configuraciones básicas de } \\
\text { computación y matemáticas, a la vez } \\
\text { que practican estrategias para el diseño, } \\
\text { resolución de problemas y otras formas de } \\
\text { colaboración entre pares. }\end{array}$ \\
\hline
\end{tabular}




\begin{tabular}{|c|c|c|}
\hline Autores & Énfasis & Perspectiva educativa \\
\hline Brennan (2011) & $\begin{array}{c}\text { Dimensiones } \\
\text { (Operacionalización } \\
\text { del término) }\end{array}$ & $\begin{array}{l}\text { La definición operacional provee un marco } \\
\text { de trabajo y vocabulario sobre pensamiento } \\
\text { computacional que puede extenderse hacia } \\
\text { educadores de etapa primaria. Sintetiza } \\
\text { un grupo de habilidades finales en los } \\
\text { estudiantes: manejo de complejidades, } \\
\text { persistencia ante los desafíos, tolerancia } \\
\text { ante la ambigüedad, habilidad para manejar } \\
\text { problemas y comunicarlos entre pares para } \\
\text { una solución común. }\end{array}$ \\
\hline $\begin{array}{c}\text { Sysło y Kwiatkowska } \\
\text { (2013) } \\
\text { Pérez y Roig-Vila } \\
\text { (2015) } \\
\text { Román (2016) } \\
\text { Zapata-Ros } \\
\text { (2019) }\end{array}$ & $\begin{array}{l}\text { Habilidades del } \\
\text { pensamiento }\end{array}$ & $\begin{array}{l}\text { El diseño instruccional del pensamiento } \\
\text { computacional deberá procurar enlazar } \\
\text { intenciones, condiciones y recursos } \\
\text { con objetivos y resultados deseados de } \\
\text { aprendizaje. En este caso con el desarrollo de } \\
\text { las habilidades que constituyen los elementos } \\
\text { del pensamiento computacional. } \\
\text { En el esquema del diseño instruccional, } \\
\text { ocupan un lugar clave las actividades. } \\
\text { Sin actividades no hay aprendizajes, y es } \\
\text { haciendo como se aprende. }\end{array}$ \\
\hline $\begin{array}{c}\text { Raja (2014) } \\
\text { Plan Ceibal (2017) } \\
\text { Llorens et al. (2017) } \\
\text { Shute et al. (2017) } \\
\text { Maris (2019) }\end{array}$ & Visión / Paradigma & $\begin{array}{l}\text { Los estudiantes que aprenden pensamiento } \\
\text { computacional a través de planes de estudios, } \\
\text { pueden comenzar a ver una relación entre las } \\
\text { asignaturas académicas, así como entre la } \\
\text { vida dentro y fuera del salón de clases. Por lo } \\
\text { tanto, añadir el pensamiento computacional } \\
\text { a la capacidad analítica del niño desde } \\
\text { la escuela, le servirá para generar otras } \\
\text { capacidades. }\end{array}$ \\
\hline $\begin{array}{l}\text { Basogain et al. (2015) } \\
\text { Cummins (2016) } \\
\text { García-Peñalvo et al. } \\
(2016)\end{array}$ & $\begin{array}{l}\text { Método / } \\
\text { Metodología }\end{array}$ & $\begin{array}{l}\text { El pensamiento computacional es un método } \\
\text { comprobado por investigadores de nivel, es } \\
\text { fácil de enseñary divertido de aprender, por lo } \\
\text { tanto, debe incorporarse a la educación para } \\
\text { que los niños se conviertan en pensadores } \\
\text { computacionales que resuelven problemas } \\
\text { variados con métodos innovadores, siendo } \\
\text { más creadores que consumidores. }\end{array}$ \\
\hline
\end{tabular}




\begin{tabular}{|c|c|l|}
\hline Autores & \multicolumn{1}{|c|}{ Énfasis } & \multicolumn{1}{|c|}{ Perspectiva educativa } \\
\hline \multirow{2}{*}{$\begin{array}{c}\text { Ortega (2017) } \\
\text { González-González } \\
(2019)\end{array}$} & Estrategia & $\begin{array}{l}\text { Al desarrollar el pensamiento computacional, } \\
\text { se logra entender qué aspectos de un } \\
\text { problema son susceptibles de computación, } \\
\text { evaluar la coincidencia entre herramientas } \\
\text { y técnicas computacionales y un problema, } \\
\text { comprender las limitaciones y el poder de } \\
\text { las herramientas y técnicas computacionales, } \\
\text { aplicar o adaptar una herramienta o técnica } \\
\text { computacional a un nuevo uso, reconocer la } \\
\text { oportunidad de usar el cálculo de una manera } \\
\text { nueva, aplicar estrategias computacionales } \\
\text { tales como dividir y conquistar en cualquier } \\
\text { dominio. }\end{array}$ \\
\hline
\end{tabular}

Fuente: Información de la tabla 1.

Como revela la tabla 3, la mayoría de las definiciones hacen énfasis en el pensamiento computacional como un proceso mental. En este proceso, el pensador computacional formula interrogantes que le permiten operacionalizar los problemas y darles solución mediante estrategias basadas en el análisis y construcción de algoritmos, y en las cuales aplica sus habilidades y herramientas mentales.

Pese a la presencia de elementos coincidentes entre conceptos, existen también aspectos disímiles que han nutrido el debate sobre el pensamiento computacional, cuya inconsistencia entre acuerdos para una definición clara del término se ha prolongado durante varios años. Parte de la diatriba desarrollada entre expertos, en ocasiones insinúa la moción de que el pensamiento computacional fue estipulado por profesionales de la computación, profesores de informática o científicos en el campo, pero recientemente se expresa la necesidad de atender el término desde cualquier contexto y se extiende la idea de saber programar o diseñar algoritmos, así como al estudio de estas ramas, a su comprensión y puesta en práctica, lo cual podría resultar idílico, no en cuanto a producir sistemas, sino para pensar de forma diferente.

Al respecto, Denning (2017) ha criticado las definiciones del pensamiento computacional que se han extendido desde las posturas de Wing debido a que éstas parecen deambular en una constante pretensión por involucrar el uso de la informática en todos los campos de saber; y a raíz de ello se ha generado una proliferación de afirmaciones que conducen a promesas exageradas sobre la utilidad del pensamiento computacional en la solución de cualquier tipo de problema real. Ante esto, es importante figurarse que se trata de una configuración mental, sobre la forma en la que se piensa, por tanto, su aplicabilidad llega a ser amplia y variable.

Ciertamente, este pensamiento no se limita a la codificación de sistemas, el enfoque del pensamiento computacional es beneficioso para usuarios que no estén necesariamente adscritos a la labor tecnológica, o efectúen operaciones mediante el uso de equipos de cómputo; este pensamiento aplica para la adquisición de habilidades 
particularmente inclinadas hacia la lógica y abstracción de datos, se procesan mentalmente las entradas de un medio, para transformarlos en salidas válidas; sin embargo, en última instancia, la aplicación del pensamiento computacional para resolver problemas o crear soluciones también sirve para programar un computador, con la intención de generar resultados deseados dentro de aquella gama de carreras que involucran el manejo de la tecnología.

Frente a este planteamiento, una de las mejores opciones para trabajar el pensamiento computacional, es analizar su incorporación en el currículo escolar obligatorio, y no solo esto, hacerlo desde etapas o niveles tempranos del sistema educativo, tal como se ha propuesto en Estados Unidos (Valverde-Berrocoso et al., 2015); al igual que en Reino Unido, España y parte del continente Europeo en general, con la afiliación de asignaturas de programación informática o manejo de tecnología, sin embargo, con frecuencia estos intentos dan por sentado que a través de la enseñanza de la programación, los niños desarrollarán plenamente el pensamiento computacional, idea que está distanciada de la realidad, porque aunque al aprender programación un estudiante desarrolla procesos cognitivos que también coinciden en las tareas de un pensador computacional, en ocasiones los cursos de programación se dedican a desarrollar estrategias donde el estudiantado entienda y aplique pensamiento para programar (Ortega, 2017).

Entonces, la idea de utilizar lenguajes de programación, sesiones prácticas con los niños en los laboratorios de computación, y las referencias para programar sistemas, resulta atractiva y pertinente para trabajar aspectos cognitivos, lógica y representación de datos con los estudiantes, pero ese ingrediente computacional ha de solicitar perentoriamente normas y directrices para su praxis dentro y fuera del aula, tal como lo establece Zapata-Ros (2015), porque en la actualidad se percibe una tendencia en la que los profesores de este campo, únicamente centran su ejercicio a la enseñanza de fundamentos de programación, donde solo se escriben líneas de código que en algunas circunstancias incluso, no son sometidas a un debate reflexivo sobre cómo intervienen los datos ingresados y las salidas generadas durante una depuración.

$\mathrm{Al}$ respecto, Adell et al. (2019) plantean que lo ideal, más que la inclusión de una asignatura específica acerca de computación o informática, es la integración del pensamiento computacional como un eje transversal del currículo en otras asignaturas, ya que la mayoría de ellas se concentran en la enseñanza de contenido dejando de lado el desarrollo de habilidades y destrezas, incluso en esta área la cual reúne un grupo de competencias muy demandadas en el mundo de hoy (Roig-Vila y Moreno-Isac, 2020).

En este orden de ideas, al recabar los planteamientos extraídos desde la revisión de documentos, la importancia de considerar el pensamiento computacional como competencia elemental para todos los sujetos, puede justificarse desde varios puntos de vista. Por una parte, desde una mirada económica, la creciente necesidad de contar con programadores y desarrolladores de sistemas para satisfacer las demandas del 
mercado laboral presente y futuro, es una inalterable tendencia, por ello, la idea de conocer tecnologías, herramientas y entornos de desarrollo desde las escuelas, debe ser evaluada por aquellas latitudes en las que aun el componente tecnológico no se incluye en la enseñanza obligatoria. La dinámica económica y social en vigencia, permite develar nuevas categorías de empleos que hace unos pocos años atrás, simplemente no existían; hoy en día se evidencia cómo el teletrabajo, la aplicación a trabajos independientes a través de Internet, suscripción a comunidades virtuales, redes sociales y tecnologías en general, mantienen a los usuarios interconectados conjuntamente con las transformaciones del campo y se expone un panorama en el que, especialmente, el conocimiento viene a ser el recurso más valioso de todos.

Además, la vanguardia tecnológica, el empleo de entornos digitales, simuladores, proyecciones y en fin, el creciente atractivo de ciertas áreas de las ciencias exactas, han dado origen al término STEM, acrónimo de Science, Technology, Engineering and Mathematics (Ciencia, Tecnología, Ingeniería y Matemáticas), es una expresión de impresa reputación hoy en día, tanto para activistas tecnológicos como organizaciones globales, dado que hace referencia a las áreas del conocimiento en las que trabajan los ingenieros y científicos, lo que forma parte de una llamativa oferta que va en absoluto ascenso para profesionales del futuro.

Equivalentemente, es imperioso que la demanda laboral de desarrolladores talentosos, se ubique en aquellas personas capaces de conocer y cumplir con los estándares de calidad de los sistemas o productos lógicos, por lo tanto, los pensadores deben contar con destrezas cognitivas que incorporen agudeza en cuanto a la abstracción, generalización y razonamiento lógico, para generar producciones de calidad, reutilizables, que sigan metodologías para el trabajo coordinado, de ágil refactorización y código limpio. La base del pensamiento computacional desde la perspectiva de Wing, reside también en el proceder minucioso y en la rigurosidad de un método científico.

Adicionalmente, desde una visión educativa, el pensamiento computacional como elemento integrante de la nueva perspectiva de alfabetización digital para todos, añade un punto fuerte en el marco de las habilidades mínimas que se requieren en la sociedad del siglo XXI, el carácter instruccional de este tipo de pensamiento transita entre las ciencias agógicas, no solo en pedagogía para este caso. Como lo declara Aparicio (2018), la tendencia de incorporar contenidos sobre tecnología y pensamiento computacional en las salas de educación primaria y secundaria ha sido desarrollada desde 2012 en Europa, con soporte en los miembros de la comunidad científica quienes defienden la introducción de estas habilidades mentales, incluso como asignaturas equiparables a las Matemáticas o la Lengua. De forma tácita es evidente que, desde el plano educativo, el ideal persigue el fortalecimiento del saber universal, donde los individuos sean iluminados y se permitan adquirir conocimientos, transformarlos con coherencia y difundirlos nuevamente para las próximas generaciones. 
En este particular, es cierto que ante la carencia de acuerdos entre los teóricos respecto al pensamiento computacional y sus elementos constituyentes, se dificulta la planeación de estrategias coordinadas para el abordaje integral de este pensamiento, ya que se deben superar barreras conceptuales relacionadas al cómo enseñar a pensar computacionalmente y cómo evaluar estos resultados; sin embargo, la potencialidad ante el tema sigue en vanguardia así como la curiosidad por mantener una cultura informatizada en la sociedad y el avance del conocimiento, lo que es propio de esta era en revolución.

\section{CONSIDERACIONES FINALES}

Sobre la base de las experiencias formativas gestionadas por Papert desde los años sesenta, se exponía ya en esa época el dilatado beneficio que tienen los conocimientos técnicos para la escritura de programas de computadoras por los estudiantes, y más aún, si se les enseñan estas destrezas mentales desde temprana edad. Las publicaciones de Papert (1980) apelan a una idea en la que, la programación informática puede coadyuvar a la articulación de un trabajo mental en el aprendiz. Los eventos ejecutados por el procesador de un computador, permiten almacenar datos, efectuar cálculo aritmético y lógico, controlar periféricos, y entre muchas otras cosas, velar por la efectividad de uso de un sistema completo. Cuando se computa, se procesan entradas y se disponen soluciones factibles ante las interrogantes o problemáticas; y desde esta particular analogía, es dicha interacción entre los elementos de una realidad que se desarrolla y vigoriza el pensamiento.

Con base en lo anterior, es vital que los estudiantes desarrollen su pensamiento computacional, pues a través de este tendrían la capacidad para entender qué aspectos de un problema son susceptibles de computación, evaluar la coincidencia entre herramientas y técnicas computacionales y un problema, comprender las limitaciones y el poder de las herramientas y técnicas computacionales, aplicar o adaptar una herramienta o técnica computacional a un nuevo uso, reconocer la oportunidad de usar el cálculo de una manera nueva, aplicar estrategias computacionales tales como dividir y conquistar en cualquier dominio, las cuales son capacidades distinguidas en la sociedad del siglo XXI.

Por otra parte, el desarrollo del pensamiento computacional dentro del plan de estudios favorece el desarrollo integral del estudiante, por lo cual será capaz de encontrar la relación entre las distintas asignaturas que cursa y la utilidad que las competencias en las cuales estas se enfocan tendrán en su vida escolar y extraescolar, en el presente y el futuro. 


\section{REFERENCIAS}

Adell, J., Llopis, M., Esteve, F., y Valdeolivas, M. (2019). El debate sobre el pensamiento computacional en educación. RIED. Revista Iberoamericana de Educación a Distancia, 22(1). https://doi. org/10.5944/ried.22.1.22303

Aho, A. (2012). Computation and Computational Thinking. The Computer Journal, 55(7), 832-835. https://doi. org/10.1093/comjnl/bxso74

Aparicio, M. (2018). Desarrollo del pensamiento computacional mediante programación en alumnos con necesidades específicas de apoyo educativo (ANEAE). [Tesis de Máster], Universidad Politécnica de Madrid. Archivo Digital UPM. http://oa.upm. es/53156/

Barr, V., y Stephenson, C. (2011). Bringing computational thinking to K-12: What is Involved and What is the role of the computer science education community? ACM Inroads, 2(1), 48-54. http://doi. org/10.1145/1929887.1929905

Basogain, X., Olabe, M., y Olabe, J. (2015). Pensamiento computacional a través de la Programación: Paradigma de Aprendizaje. RED Revista de Educación a Distancia, 46(6). http://www.um.es/ead/red/46. https://doi.org/10.6018/red/46/6

Brennan, K. (2011). Computational Thinking Concepts [Webinar]. ScratchEd Team. http://scratched.gse.harvard. edu/resources/computational-thinkingconcepts-march-2011-webinar.html

Codelearn. (4 de noviembre de 2019). ¿Qué es el pensamiento computacional? Codelearn. https://codelearn. es/beneficios-del-pensamientocomputacional/

Computing at School. (2015). Computational thinking. A guide for teachers. http://computingatschool.org.uk/ computationalthinking
Cummins, K. (May 25, 2016). Five reasons why computational thinking is an essential tool for teachers and students. Edgalaxy.com - Cool stuff for nerdy teachers. https://www.edgalaxy.com/ journal/2016/5/25/five-reasons-whycomputational-thinking-is-an-essentialtool-for-teachers-and-students

Cuny, J., Snyder, L., y Wing, J. M. (2010). Demystifying computational thinking for non-computer scientists. Unpublished manuscript, referenced in http://www. cs.cmu.edu/ CompThink/resources/ TheLinkWing.pdf

Denning, P. (2017). Remaining trouble spots with computational thinking. Communications of the ACM, 6o(6), 3339. https://doi.org/10.1145/2998438

Formación en Red del INTEF. (s.f.). ¿Qué es el pensamiento computacional? http://formacion.intef.es/pluginfile. $\mathrm{php} / 87694 / \mathrm{mod} \mathrm{imscp} /$ content $/ 9 / \mathrm{qu}$ es el pensamiento computacional.html

García-Peñalvo, F. J. (2016). What computational thinking is. Journal of Information Technology Research, 9(3), v-viii. https://repositorio.grial.eu/ bitstream/grial/679/1/CT.pdf

García-Peñalvo, F., Reimann, D., Tuul, M., Rees, A., y Jormanainen, I. (2016). An overview of the most relevant literature on coding and computational thinking with emphasis on the relevant issues for teachers. Belgium: TACCLE3 Consortium. http://doi.org/10.5281/zenodo.165123

González-González, C. (2019). State of the art in the teaching of computational thinking and programming in childhood education. Education in the Knowledge Society, 2O(17). https://doi.org/10.14201/ \begin{tabular}{lll} 
eks2019 $20 \quad$ a17 \\
\hline
\end{tabular}

International Society for Technology in Education ISTE \& Computer Science Teachers Association CSTA. (2011). Operational Definition of Computational 
Thinking for K-12 Education. http:// www.iste.org/docs/ct-documents/ctleadershipt-toolkit.pdf?sfvrsn $=4$.

Llorens, F., García-Peñalvo, F., Molero, X., y Vendrell, E. (2017). La enseñanza de la informática, la programación y el pensamiento computacional en los estudios preuniversitarios. Education in the Knowledge Society, 18(2), 7-17. https://doi.org/10.14201/eks2017182717

Mannila, L., Dagiene, V., Demo, B., Grgurina, N., Mirolo, C., Rolandsson, L., y Settle, A. (2014). Computational Thinking in K-9 Education. Proceedings of the Working Group Reports of the 2014 on Innovation \& Technology in Computer Science Education Conference (1-29). New York, USA: ACM. https://doi. org/10.1145/2713609.2713610

Maris, S. (2019). Pensamiento computacional: por qué incluirlo en el proceso de aprendizaje. Net-Learning. https://www.net-learning.com.ar/ blog/herramientas/pensamientocomputacional-por-que-incluirlo-en-elproceso-de-aprendizaje.html

Ortega, B. (2017). Pensamiento computacional $y$ resolución de problemas. [Tesis doctoral], Universidad Autónoma de Madrid. Repositorio UAM. https://repositorio.uam.es/bitstream/ handle/10486/683810/ortega ruiperez beatriz.pdf?sequence $=1$ \&isAllowed $=y$

Papert, S. (1980). Mindstorms: Children, computers, and powerful ideas. Basic Books Inc. http://www.arvindguptatoys. com/arvindgupta/mindstorms.pdf

Pérez, H., y Roig-Vila, R. (2015). Entornos de programación no mediados simbólicamente para el desarrollo del pensamiento computacional. Una experiencia en la formación de profesores de Informática de la Universidad Central del Ecuador. RED Revista de Educación a Distancia, 46, 1-22. https://dialnet.unirioja.es/servlet/ articulo?codigo $=6022698$
Plan Ceibal. (13 de diciembre de 2017). ¿Qué aporta al aula el Pensamiento Computacional? Plan Ceibal. https:// www.ceibal.edu.uy/es/articulo/ que-aporta-al-aula-el-pensamientocomputacional

Raja, T. (June 16, 2014). Is Coding the New Literacy? Mother Jones. http://www. motherjones.com/media/2014/06/ computer-science-programming-codediversity-sexism-education

Rodríguez, S. (2018). ¿Qué es el pensamiento computacional? Blog Edikeus.com. https://edikeus.com/que-es-elpensamiento-computacional/

Roig-Vila, R., y Moreno-Isac, V. (2020). El pensamiento computacional en educación. Análisis bibliométrico y temático. RED. Revista de Educación a Distancia, 20(63). http://dx.doi. org/10.6018/red.402621

Román, M. (2016). Codigoalfabetización y pensamientocomputacionalenEducación Primaria y Secundaria: validación de un instrumento y evaluación de programas. [Tesis Doctoral], Universidad Nacional de Educación a Distancia. http://e-spacio. uned.es/fez/eserv/tesisuned:EducacionMroman/ROMAN GONZALEZ Marcos Tesis.pdf

Selby, C., y Woollard, J. (2013). Computational thinking: the developing definition. UK: University of Southampton E-prints. https://eprints. soton.ac.uk/356481

Shute, V., Dun, C., y Asbell-Clarke, J. (2017). Demystifying computational thinking. Educational Research Review, 22, 142-158. https://doi.org/10.1016/j. edurev.2017.09.003

Snalune, P. (2015). The Benefits of Computational Thinking. ITNOW, 57(4), 58-59. https://doi.org/10.1093/itnow/ bwv111

Sysło, M., y Kwiatkowska, A. (2013). Informatics for All High School Students: A Computational Thinking Approach. 
En I. Diethelm y R. Mittermeir (Eds.), Informatics in Schools. Sustainable Informatics Education for Pupils of all Ages. (43-56). https://doi. org/10.1007/978-3-642-36617-8 4

The Royal Society (2012). Shut down or restart? The way forward for computing in UK schools. Computing in Schools Reports. https://royalsociety.org/topicspolicy/projects/computing-in-schools/ report/

Valverde-Berrocoso, J., Fernández-Sánchez, M., y Garrido-Arroyo, M. (2015). El pensamiento computacional y las nuevas ecologías del aprendizaje. RED Revista de Educación a Distancia, 46(3), 1-18. https://doi.org/10.6018/red/46/3

Wing, J. (2006). Computational Thinking. Communications of the ACM,
49(3), 33-35. https://www.cs.cmu. edu/ 15110-s13/Wingo6-ct.pdf. https:// doi.org/10.1145/1118178.1118215

Wing, J. (2010). Computational Thinking: What and Why? https://www. cs.cmu.edu/ CompThink/resources/ TheLinkWing.pdf

Zapata-Ros, M. (2015). Pensamiento computacional: una nueva alfabetización digital - Computational thinking a new digital literacy. RED Revista de Educación a Distancia, 46(4) https://www.um.es/ ead/red/46/zapata.pdf. https://doi. org $/ 10.6018 / \mathrm{red} / 46 / 4$

Zapata-Ros, M. (2019). Computational Thinking Unplugged. Education in the Knowledge Society, 20(18). https://doi. org/10.14201/eks2019 $20 \quad$ a18

\section{PERFIL ACADÉMICO Y PROFESIONAL DE LAS AUTORAS}

Norailith Polanco-Padrón. Profesora Asistente de la Universidad del Zulia, Núcleo Costa Oriental del Lago, Programa de Humanidades y Educación Licenciada en Educación, mención Informática. Máster en Sistemas de Telecomunicaciones. Participante del Doctorado en Ciencias de la Educación, Universidad del Zulia, Núcleo Costa Oriental del Lago. Investigador PEII nivel A-1. Líneas de trabajo: Educación y tecnología, Informática, Telemática, Telecomunicaciones, Educación virtual. https://orcid.org/0000-0002-9436-8177

E-mail: norailithp@gmail.com

Sonia Ferrer-Planchart. Profesora Asociada de la Universidad del Zulia, Núcleo Costa Oriental del Lago. Licenciada en Educación, Mención Ciencias Pedagógicas, Área Orientación. Magister Scientiarum en Orientación Educativa. Doctora en Educación por la Universidad de Córdoba, España. Coordinadora del Centro de Orientación de la Universidad del Zulia Núcleo Costa Oriental del Lago. Líneas de trabajo: Educación y desarrollo humano, Inclusión, Orientación vocacional, Tecnología Educativa. https://orcid.org/000o-0001-7886-2821

E-mail: soniaferrerp4053@gmail.com 
Mariana Fernández-Reina. Profesora Titular de la Universidad del Zulia, Núcleo Costa Oriental del Lago. Licenciada en Educación, Mención Ciencias Pedagógicas, Área Orientación. Magister Scientiarum en Orientación Educativa. Doctora en Educación por la Universidad de Córdoba, España. Líneas de trabajo: Educación y desarrollo humano, Neurociencias, Orientación académica, Orientación vocacional, Tecnología educativa, Innovación educativa, Educación virtual. https:// orcid.org/0000-0003-2486-8392

E-mail: mfernandezreina@gmail.com

Dirección:

Universidad del Zulia

Núcleo Costa Oriental del Lago

Av. Universidad, frente al Palacio Arzobispal.

Cabimas, estado Zulia, Venezuela.

Fecha de recepción del artículo: 03/05/2020

Fecha de aceptación del artículo: 19/08/2020

Fecha de aprobación para maquetación: 17/09/2020 